\title{
Validation and Divergence of the Activation Energy Barrier Crossing Transition at the AOT/Lecithin Reverse Micellar Interface
}

\author{
S. Shankara Narayanan, Sudarson Sekhar Sinha, Rupa Sarkar, and Samir Kumar Pal* \\ Unit for Nano Science \& Technology, Department of Chemical, Biological \& Macromolecular Sciences, \\ S. N. Bose National Centre for Basic Sciences, Block JD, Sector III, Salt Lake, Kolkata 700 098, India
}

Received: October 18, 2007; In Final Form: January 1, 2008

\begin{abstract}
In this report, the validity and divergence of the activation energy barrier crossing model for the bound to free type water transition at the interface of the AOT/lecithin mixed reverse micelle (RM) has been investigated for the first time in a wide range of temperatures by time-resolved solvation of fluorophores. Here, picosecondresolved solvation dynamics of two fluorescent probes, ANS (1-anilino-8-naphthalenesulfonic acid, ammonium salt) and Coumarin 500 (C-500), in the mixed RM have been carefully examined at 293, 313, 328, and 343 $\mathrm{K}$. Using the dynamic light scattering (DLS) technique, the size of the mixed RMs at different temperatures was found to have an insignificant change. The solvation process at the reverse micellar interface has been found to be the activation energy barrier crossing type, in which interface-bound type water molecules get converted into free type water molecules. The activation energies, $E_{\mathrm{a}}$, calculated for ANS and C-500 are 7.4 and $3.9 \mathrm{kcal} \mathrm{mol}^{-1}$, respectively, which are in good agreement with that obtained by molecular dynamics simulation studies. However, deviation from the regular Arrhenius type behavior was observed for ANS around $343 \mathrm{~K}$, which has been attributed to the spatial heterogeneity of the probe environments. Time-resolved fluorescence anisotropy decay of the probes has indicated the existence of the dyes in a range of locations in RM. With the increase in temperature, the overall anisotropy decay becomes faster revealing the lability of the microenvironment at elevated temperatures.
\end{abstract}

\section{Introduction}

The interface of water and surfactant polar head group in micelles and reverse micelles (RMs) offers a large commonality with the interfaces formed by biological macromolecules. The dynamics of water at the interfaces of such systems are nearly $2-3$ orders of magnitude slower than that of the bulk water, ${ }^{1,2}$ and this slow solvation dynamics play an important role in many natural biological processes like electron transfer, ion transport, and molecular recognition in the hydrophobic cavities of proteins and membrane lipid bilayers..$^{3,4}$ The origin of this slow component is due to a dynamic equilibrium between the bound and free water molecules; the former one is assumed to form hydrogen bonds with the polar head groups of surfactants, while the latter one retains bulk type behavior. ${ }^{5}$ This dynamic equilibrium is extremely sensitive to changes in the external environments such as temperature, ${ }^{6}$ pressure, ${ }^{7}$ additives, ${ }^{8}$ and so forth, since it can induce changes in different physicochemical properties of RMs. Thus, it is important to study the effect of different external conditions on the solvation dynamics of water in a restricted environment.

In the limit of very high binding energy $\left(E_{\mathrm{a}}\right)$, the slow component of solvation $\left(\tau_{\text {solv }}\right)$ can be expressed as ${ }^{9-11}$

$$
\frac{1}{\tau_{\text {solv }}} \approx k_{\mathrm{bf}}=\frac{k_{\mathrm{B}} T}{h} \mathrm{e}^{-E_{\mathrm{a}} / R T}
$$

where $k_{\mathrm{bf}}$ denotes the rate constant and $E_{\mathrm{a}}$ the activation energy for bound to free water transition, respectively. The validity of this activation energy barrier crossing transition of bound to

* Corresponding author. E-mail: skpal@bose.res.in, Fax: 91332335 3477 . free water at the micellar interface has been investigated in some of the recent literature. ${ }^{9,12}$ A gradual increase in the solvation rate of 4-aminopthalamide (4-AP) in TX-100 micelle with the increase in temperature has been reported by Sen et al. ${ }^{9}$ and explained on the basis of the enhancement of the activationcontrolled exchange of bound to free water transition. On the other hand, temperature-dependent solvation dynamics of Coumarin 153 (C-153) and Coumarin 151 (C-151) in Triton-X-100 and Brij-35 micelles have been reported by Kumbhakar et al., ${ }^{12}$ which showed an unusual inversion around $298 \mathrm{~K}$ for Triton$\mathrm{X}-100$ but revealed a nominal temperature effect on the solvation dynamics for Brij-35. This irregular trend of the solvation dynamics has been elucidated by Kumbhakar et al. ${ }^{12}$ in terms of hydration number and size of the micelle. According to them, this difference in the solvation behavior is due to the nature of the probes used and their location in the micelle. The same group has recently reported $^{13}$ the temperature-dependent solvation dynamics of coumarin dyes in aqueous triblock copolymer micelles in the temperature range 293-343 K and interpreted their results in view of structural and hydration studies. Temperature-dependent solvation dynamics of a fluorophore DCM (4-(dicyanomethylene)-2-methyl-6( $p$-(dimethylamino)styryl)-4H-pyran) in SDS micelle have been recently reported from our group, ${ }^{14}$ and the study revealed a good agreement with the Arrhenius type behavior within the framework of overall structural integrity of the micelle.

The role of size and hydration of ionic/nonionic RM in the studies of relaxation dynamics of water has been established in a number of publications. ${ }^{15-19}$ Mid-infrared ultrafast pumpprobe spectroscopy of RMs of different well-defined sizes revealed slow vibrational relaxation dynamics of water at the interface. ${ }^{16}$ Using the picosecond-resolved fluorescence spec- 
troscopic technique, Bhattacharyya and co-workers ${ }^{15}$ have reported the nanosecond relaxation of water in the AOT RM. However, to date very little attention has been paid to track the effect of the temperature on the mobility of water molecules in the RMs. ${ }^{6,20,21}$ In this regard, the validation of Arrhenius type behavior at the surface of AOT-RM of various degrees of hydration $\left(w_{0}\right)$ has been reported from our group. ${ }^{6,21}$ In this study, we have investigated the temperature-dependent reorganization of entrapped water molecules at the interface of AOT/ lecithin mixed RM in a wide temperature range 293-343 K. The presence of cosurfactants in mixed surfactant systems often gives rise to enhancement of solubilization capacity by altering the interfacial rigidity of the RM. ${ }^{22}$ To explore the temperature effect on the solvation dynamics in RM systems, we have used two fluorescent probes, ANS (1-anilino-8-naphthalenesulfonic acid, ammonium salt) and Coumarin 500 (C-500). The most probable location of ANS is close to the reverse micellar interface. ${ }^{23}$ It should be noted that the probe ANS is expected to have less affinity toward AOT head groups compared to that of the lecithin as both ANS and AOT are anionic in nature. The probe $\mathrm{C}-500$ being neutral in nature should have closer approach toward the interface of the RM. The structural integrity of the RM in the temperature range $293-343 \mathrm{~K}$ has been examined by the dynamic light scattering (DLS) technique. Time-resolved anisotropy study has been carried out to explore the location of the probe at different temperatures. Our studies attempt to explore the conditions for the validation of Arrhenius type behavior in the temperature-dependent solvation dynamics at the RM interface.

\section{Materials and Methods}

Chemicals are obtained from the following sources: sodium bis(2-ethylhexyl)sulfosuccinate (AOT, Sigma); L- $\alpha$-phosphatidylcholine (lecithin, Sigma); 2,2,4-trimethylpentane (isooctane, Sigma); Coumarin 500 (C-500, Exciton, Figure 1a); ANS (1anilino-8-naphthalenesulfonic acid, ammonium salt, Sigma, Figure 1a). The chemicals are of highest commercially available purity and used as received. All aqueous solutions were prepared with deionized water from Millipore system. AOT and lecithin are dissolved in isooctane to prepare $100 \mathrm{mM}$ total surfactant concentration, and the required amount of water is injected to obtain the degree of hydration $w_{0}=10\left(w_{0}=\right.$ [water] [surfactant]). Small-angle neutron scattering (SANS) ${ }^{24}$ and transmission electron microscopy (TEM) ${ }^{25}$ studies reveal the spherical shape of RM at [AOT]:[lecithin] = 3:1. Temperaturedependent steady-state emission is measured with a Jobin Yvon Fluoromax-3 fluorometer with a temperature-controller attachment from Julabo (model F32). DLS measurements are done with a Nano S Malvern instrument employing a $4 \mathrm{~mW} \mathrm{He}-\mathrm{Ne}$ laser $(\lambda=632.8 \mathrm{~nm})$ equipped with a thermostated sample chamber. All the scattered photons are collected at a $173^{\circ}$ scattering angle. The scattering intensity data are processed using the instrumental software to obtain the hydrodynamic diameter $\left(d_{\mathrm{H}}\right)$ and the size distribution of the scatterer in each sample. The instrument measures the time-dependent fluctuation in the intensity of light scattered from the particles in solution at a fixed scattering angle. The hydrodynamic diameter $\left(d_{\mathrm{H}}\right)$ of the RMs is estimated from the intensity autocorrelation function of the time-dependent fluctuation in intensity. $d_{\mathrm{H}}$ is defined as

$$
d_{\mathrm{H}}=\frac{k_{\mathrm{B}} T}{3 \pi \eta D}
$$

where $k_{\mathrm{B}}$ is the Boltzmann constant, $\eta$ is the viscosity, $D$ is the (a)<smiles>CCNc1ccc2c(C(F)(F)F)cc(=O)oc2c1</smiles>

Coumarin 500 (Probe)

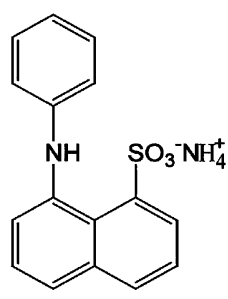

ANS (Probe) (b)

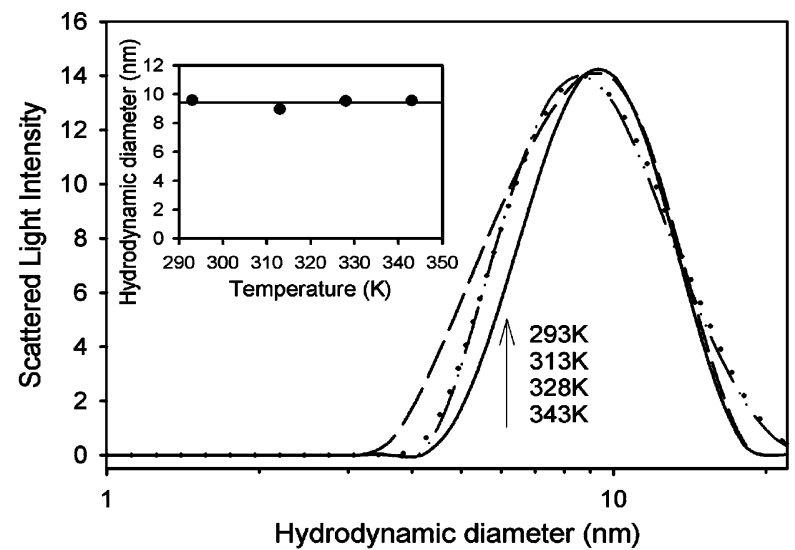

Figure 1. (a) Molecular structure of the probes Coumarin 500 (C500) and ANS. (b) Scattered light intensity distribution graph of the DLS experiment on $w_{0}=10 \mathrm{AOT} /$ lecithin RMs in the temperature range 293-343 K. The inset shows the variation of the hydrodynamic diameter $\left(d_{\mathrm{H}}\right)$ of RMs as a function of temperature. The solid line indicates the average diameter of RM to be $9.4 \mathrm{~nm}$.

translational diffusion coefficient, and $T$ is the absolute temperature. In a typical size distribution graph from the DLS measurement, the $x$-axis shows a distribution of size classes in nanometers, while the $y$-axis shows the relative intensity of the scattered light.

Fluorescence transients have been measured and fitted by using the commercially available spectrophotometer (LifeSpecps) from Edinburgh Instruments (Livingston), U.K. (excitation wavelengths of 375 and $409 \mathrm{~nm}$ with $75 \mathrm{ps}$ instrument response function (IRF) have been used to excite ANS and C-500, respectively) with an attachment for temperature-dependent studies (Julabo, F32). The observed fluorescence transients are fitted by using a nonlinear least-square fitting procedure to a function $\left(X(t)=\int_{0}^{t} E\left(t^{\prime}\right) R\left(t-t^{\prime}\right) \mathrm{d} t^{\prime}\right)$ comprising of convolution of the IRF $(E(t))$ with a sum of exponentials $(R(t)=A+$ $\left.\sum_{i=1}^{N} B_{i} \mathrm{e}^{-t / \tau_{i}}\right)$ with preexponential factors $\left(B_{i}\right)$, characteristic lifetimes $\left(\tau_{i}\right)$, and a background $(A)$. Relative concentration in a multiexponential decay is finally expressed as $a_{n}=B_{n} /($ $\left.\sum_{i=1}^{N} B_{i}\right)$. The quality of the curve fitting is evaluated by reduced $\chi^{2}$ and residual data.

To construct time-resolved emission spectra (TRES), we follow the technique described in refs 26 and 27 . The timedependent fluorescence Stokes' shifts, as estimated from TRES, are used to construct the solvation correlation function $C(t)$ and are defined as

$$
C(t)=\frac{v(t)-v(\infty)}{v(0)-v(\infty)}
$$

where $v(0), v(t)$, and $v(\infty)$ are the emission maxima $\left(\right.$ in $\mathrm{cm}^{-1}$ ) at times $0, t$, and infinity, respectively. The $v(\infty)$ values have been taken to be the emission frequency beyond which insignificant or no spectral shift is observed. The $C(t)$ function represents the temporal response of the solvent relaxation 
(a)
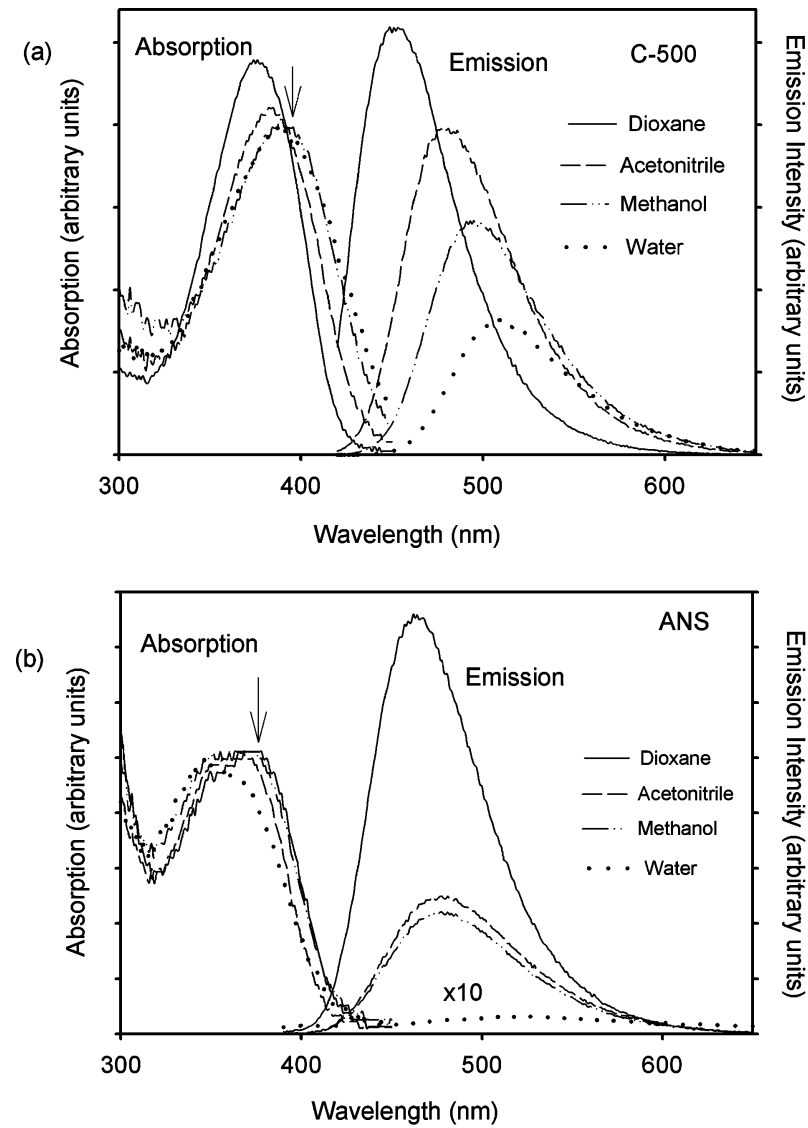

Figure 2. (a) Steady-state absorption and fluorescence spectra of (a) C-500 and (b) ANS in 1,4-dioxane, acetonitrile, methanol, and water. The emission spectrum of ANS in water is 10 times magnified for comparison. An arrow marks the excitation wavelength used to excite the probes $\left(\lambda_{\mathrm{ex}}=375 \mathrm{~nm}\right.$ for ANS and $409 \mathrm{~nm}$ for C-500).

process, as occurs around the probe following its photoexcitation and the associated change in the dipole moment. For anisotropy, $r(t)$ measurements, emission polarization is adjusted to be parallel or perpendicular to that of the excitation and anisotropy is defined as

$$
r(t)=\frac{\left[I_{\text {para }}-G I_{\text {perp }}\right]}{\left[I_{\text {para }}+2 G I_{\text {perp }}\right]}
$$

$G$, the grating factor, is determined by following the longtime tail matching technique. ${ }^{28}$ All the anisotropies have been measured at the emission maxima.

\section{Results and Discussion}

Figure $1 \mathrm{~b}$ shows the scattered light intensity distribution graph of the DLS experiment on $w_{0}=10 \mathrm{AOT} /$ lecithin mixed RMs in the temperature range 293-343 K. The inset of Figure 1b depicts the variation of the average hydrodynamic diameter of $w_{0}=10$ mixed RMs as a function of temperature. It is evidenced from the figures that, with the increase in temperature, the reverse micellar diameter changes marginally, and the measured hydrodynamic diameter is $9.4 \pm 0.6 \mathrm{~nm}$. Thus in this study, the structural integrity of $w_{0}=10 \mathrm{AOT} /$ lecithin mixed RMs in our experimental temperature window has been assured. C-500, a well-known solvation probe, ${ }^{6,29}$ is sparingly soluble in water and shows reasonably good solubility in nonpolar solvents like isooctane and 1,4-dioxane. Figure 2 a presents the absorption and fluorescence spectra of C-500 in four different solvents. It is seen that both the absorption and fluorescence spectra of C-500 are strongly dependent on the polarities of the solvents. The absorption peak of C-500 is blue-shifted as we move from the polar water medium to nonpolar dioxane medium, consistent with the earlier observations by Nad et al. ${ }^{30}$ and Das et al. ${ }^{31} \mathrm{~A}$ similar trend is observed in the emission spectra of C-500 in the four different solvents (Figure 2a). It should be mentioned that C-500 in isooctane could not be excited at $409 \mathrm{~nm}$. Table 1 lists the emission peak and quantum yield $(\Phi)$ for C-500 in the four different solvents. From Table 1 and Figure $2 \mathrm{a}$, it is clear that the emission from $\mathrm{C}-500$ is stronger in nonpolar solvent (dioxane) compared to those in the polar solvents (methanol and water). This indicates that the fluorescence quenching of C-500 is associated with increase in polarity and/ or hydrogen bonding (HB) ability of the solvents. A detail photophysical study of C-500 in different solvents has recently been carried by Das et al. ${ }^{31,32}$ and Nad et al. ${ }^{30}$ Our observation of spectral properties in the four different solvents is in good agreement with their studies. It should be noted that hydrogenbonding interactions have been proved to play a vital role in the electronically excited states of other fluorophores including coumarin dye (e.g. Coumarin $102^{33}$ ) in recent literature. ${ }^{34-37}$ In a recent spectroscopic study ${ }^{32}$ on the hydrogen-bonding properties of C-500, it has been demonstrated that the quantum yield of the dye C-500 in hexane (devoid of hydrogen bonding, $\Phi=0.76$ ) is comparable to those in $\mathrm{CHCl}_{3}$ (hydrogen-bond donor, $\Phi=0.81$ ) and THF (hydrogen-bond acceptor, $\Phi=$ $0.81)$. However, the emission characteristics of other coumarin dyes (e.g., C-151, C-35) are demonstrated ${ }^{32}$ to be heavily dependent on the HB properties of the solvents used. Moreover, in a recent spectroscopic study, ${ }^{31}$ it has been concluded that C-500 is less efficient in forming hydrogen bonds with the solvent than the other coumarin dye (C-151) by analyzing the absorption properties of the dyes in terms of Kamlet-Taft solvent parameters. Our experimental observation (Table 1) on the spectral characteristic of C-500 emission in dioxane (emission peak at $450 \mathrm{~nm}$ and $\Phi=0.81$ ) and acetonitrile (emission peak at $480 \mathrm{~nm}$ and $\Phi=0.64$ ), both of which do not offer HB interaction with $\mathrm{C}-500$, reveals that the solvents are expected to contribute more polaritywise than HB-wise.

In our studies we have also used another well-known solvation probe, ANS, ${ }^{38}$ widely utilized for studying protein dynamics. In contrast to C-500, ANS is completely insoluble in bulk isooctane. ANS in bulk water reveals an absorption peak at $360 \mathrm{~nm}$ with an emission maximum at $520 \mathrm{~nm}$ (Figure 2b). The steady-state absorption and emission spectra of the probe ANS in four different solvents are shown in Figure $2 b$, and its various spectral parameters in different solvents are listed in Table 1. As in the case of C-500, ANS emission also depends strongly on the polarity of the solvent. The steady-state emission of ANS is quenched dramatically in polar solvents as compared to nonpolar solvent dioxane (Figure 2b). Because of its bichromophoric structure, ANS is known ${ }^{39}$ to undergo twisted intramolecular charge transfer (TICT), which essentially depends on the polarity of the solvent. A detail molecular mechanism of fluorescence of a similar TICT probe, TNS, has been reported previously. ${ }^{40}$ In nonpolar solvents the steady-state emission is strong and is mostly from the locally excited state, i.e., before charge separation. In polar solvents, the fluorescence decreases and is dominated by emission from the TICT state. The solvent polarity and rigidity determine the wavelength and quantum yield of emission of the probe ANS (Table 1).

Figure $3 \mathrm{a}$ presents the steady-state absorption and fluorescence spectra of C-500 (excitation $\lambda=409 \mathrm{~nm}$ ) in $w_{0}=10$ 
TABLE 1: Emission Peak and Quantum Yield of ANS and C-500 in Different Systems ${ }^{a}$

\begin{tabular}{lcccc}
\hline \multirow{2}{*}{ system } & \multicolumn{2}{c}{ ANS } & \multicolumn{2}{c}{ C500 } \\
\cline { 2 - 4 } & em peak $(\mathrm{nm})$ & quantum yield & em peak (nm) & quantum yield \\
\hline 1,4-dioxane & 465 & 0.55 & 485 & 0.81 \\
acetonitrile & 478 & 0.19 & 500 & 0.57 \\
methanol & 478 & 0.16 & 510 & 0.46 \\
water & 520 & 0.004 & 495 & 0.32 \\
RM at 293 K & 495 & 0.08 & 495 & 0.45 \\
RM at 313 K & 495 & 0.06 & 495 & 0.38 \\
RM at 328 K & 495 & 0.05 & 495 & 0.32 \\
RM at 343 K & 495 & 0.04 & 0.27
\end{tabular}

${ }^{a}$ Quantum yields for the samples have been determined using proflavin in water, $\mathrm{pH}=7.0$, as the standard whose quantum yield is reported to be 0.34 .

(a)

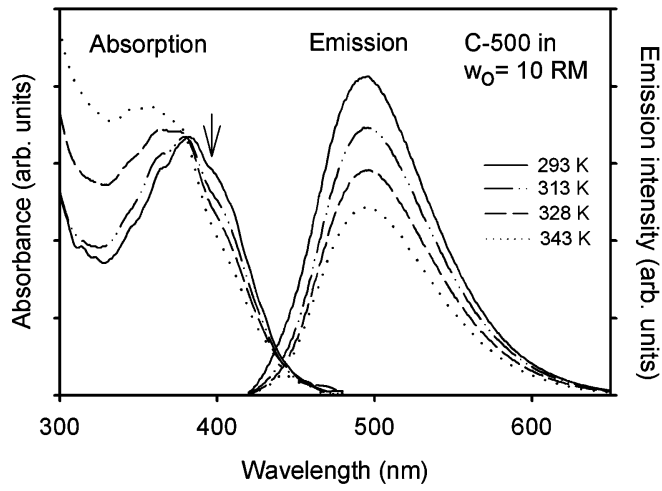

(b)

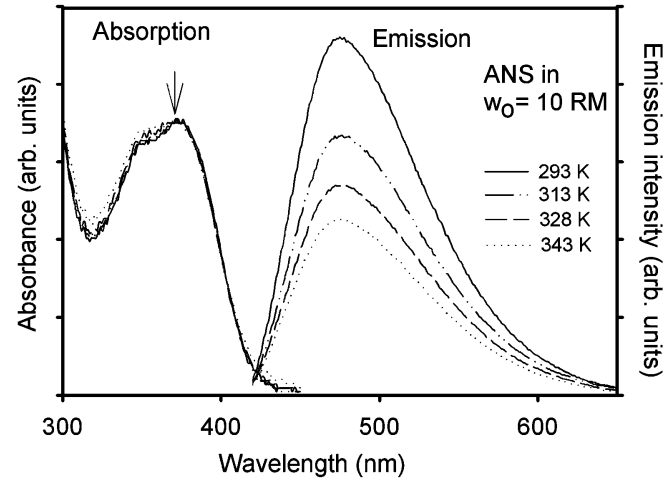

Figure 3. Steady-state absorption and fluorescence spectra of (a) C-500 and (b) ANS in $w_{0}=10$ mixed RM at 293, 313, 328, and $343 \mathrm{~K}$. An arrow marks the excitation wavelength used to excite the probes $\left(\lambda_{\mathrm{ex}}\right.$ $=375 \mathrm{~nm}$ for ANS and $409 \mathrm{~nm}$ for C-500).

$\mathrm{RM}$ in the temperature range 293-343 K. The absorption spectrum of C-500 in $w_{0}=10 \mathrm{RM}$ at $293 \mathrm{~K}$ (Figure 3a) shows a peak at $380 \mathrm{~nm}$ with a shoulder at $400 \mathrm{~nm}$ indicating a population of the probe $\mathrm{C}-500$ in the polar environment of the RM. Hence, it is clear that, at $409 \mathrm{~nm}$ excitation, only those populations of C-500 molecules are excited which are in the polar environments of the RM. ${ }^{29}$ Upon excitation of the probe at $409 \mathrm{~nm}$, its emission is found to peak at $495 \mathrm{~nm}$ (Figure 3a), confirming the location of $\mathrm{C}-500$ in the close vicinity of the RM interface. The absorption spectrum of C-500 shows a blue shift at elevated temperatures, which could reflect that the immediate environments of C-500 are becoming relatively less polar indicating dye migration from interface to the nonpolar isooctane side. However, insignificant change in the emission peak (excitation $\lambda=409 \mathrm{~nm}$ ) at elevated temperatures (Figure $3 a)$ indicates unperturbed excited-state equilibrium of the probe $\mathrm{C}-500$ in the RM. The observation is consistent with the fact that the interface-bound C-500 probe molecules are selectively excited at $409 \mathrm{~nm}$ excitation, leading to unaltered spectral shape of the probe molecules at the interface with temperature. The observation also stands against a significant difference in the spatial heterogeneity of the probe due to probe migration to different environments in the RM at elevated temperature ${ }^{41,42}$ under our experimental conditions $\left(\lambda_{\max }=409 \mathrm{~nm}\right)$. The emission spectrum of $\mathrm{C}-500$ in RM (Figure 3a) reveals quenching of fluorescence intensity with increase in temperature. It can be argued that the quantum yield of the probe increases when introduced in a restricted environment of RMs as compared to that in the bulk water (Table 1). As the temperature increases, the interface-bound water in the RMs becomes converted into free-type water (increase in environmental polarity), which in turn leads to decrease in the quantum yield of the probe ${ }^{6}$ (Figure $3 \mathrm{a}$ and Table 1). The emission peak and quantum yield of C-500 in mixed RMs at different temperatures are listed in Table 1, which clearly signifies the essential role played by environmental polarity in the quenching of C-500 fluorescence with the temperature. From our observations, it could be concluded that the change in the environmental polarity at elevated temperatures does not suffice to change the emission peaks of the probe $\mathrm{C}-500$ but is enough to contribute to the quenching of the probe emission.

The steady-state absorption and fluorescence spectra of ANS (excitation $\lambda=375 \mathrm{~nm}$ ) in $w_{0}=10 \mathrm{RM}$ in the temperature range $293-343 \mathrm{~K}$ has been presented in Figure $3 \mathrm{~b}$. Due to its anionic nature, ANS is extremely hydrophilic, and when dissolved in AOT/lecithin RM, the probe is expected to reside in the water pool of the RM. From Table 1, it is evident that the ANS emission increased in the RM followed by a blue shift of $25 \mathrm{~nm}$ at room temperature compared to that in bulk water. The observation is consistent with the fact that a significant population of the probe resides in the interfacial region of the RM, where polarity of water molecule and dynamical freedom of the probe are much lower than that in the center of the water pool. In the RM interfacial region, the anionic character of ANS is expected to make its interaction with the negatively charged head group of AOT less favorable. Thus, the probes ANS in the reversal micellar interface are expected to interact with the lecithin polar head groups. Figure $3 \mathrm{~b}$ shows quenching in the ANS emission at elevated temperatures, indicating increase in the polarity around the local environment of the interface-bound ANS at higher temperature due to activation energy barrier crossing transition of bound to free water at the reverse micellar interface. ${ }^{6,21}$ The emission peak and quantum yield of ANS in mixed RMs at different temperatures are listed in Table 1, which reveals the decrease in ANS emission at elevated temperatures. The insignificant change in the spectral shape of the absorption and fluorescence spectra of ANS in $w_{0}=10$ RM (Figure 3b) rules out the possibility of ANS migration in various environments with the increase in temperature. ${ }^{41,42}$

To investigate the alteration of dynamical properties of water at the reverse micellar interface with the change of temperature, 

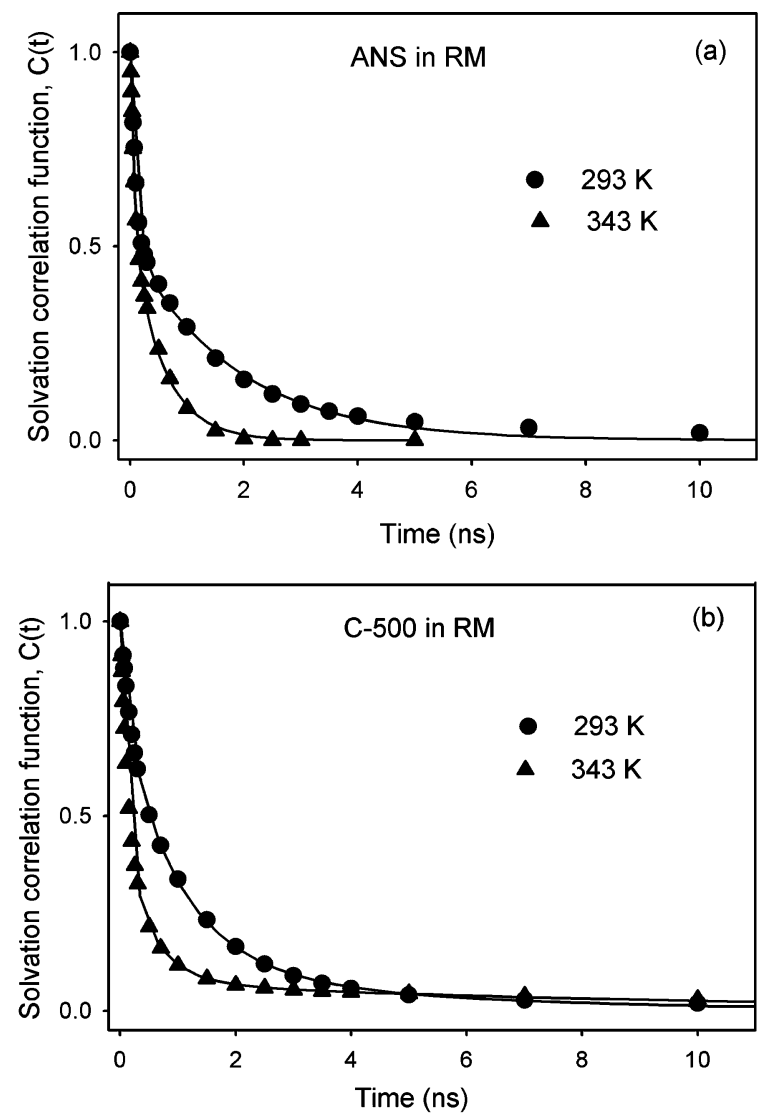

Figure 4. Solvation correlation function, $C(t)$, of (a) ANS and (b) C-500 in $w_{0}=10$ mixed RM at 293 and $343 \mathrm{~K}$. Solid lines indicate numerical fitting to the experimental data points.

TABLE 2: Decay Parameters of $C(t)$ of ANS in $w_{0}=10$ Mixed RM at Different Temperatures

\begin{tabular}{cccc}
\hline temp $(\mathrm{K})$ & $\tau_{1}\left(\mathrm{a}_{1}\right)(\mathrm{ns})$ & $\tau_{2}\left(\mathrm{a}_{2}\right)(\mathrm{ns})$ & $\left\langle\tau_{\text {solv }}\right\rangle(\mathrm{ns})$ \\
\hline 293 & $0.10(0.50)$ & $1.82(0.50)$ & 0.96 \\
313 & $0.10(0.39)$ & $1.70(0.61)$ & 1.07 \\
328 & $0.07(0.41)$ & $1.40(0.59)$ & 0.85 \\
343 & $0.07(0.45)$ & $0.55(0.55)$ & 0.33
\end{tabular}

we have examined picosecond-resolved solvation dynamics of the probes ANS and C-500 in $w_{0}=10$ RM. Figure 4a,b depicts the solvation correlation function, $C(t)$, constructed from timeresolved emission spectra (TRES) of ANS and C-500 in RM, respectively. The solvent relaxation process immediately after photoexcitation of the probe and the associated change in dipole moment are characterized by $C(t)$ decay. ANS is known to be an efficient probe to explore faster and slower solvation dynamics in restricted environments..$^{23,43}$ The $C(t)$ curves of ANS in $w_{0}=10 \mathrm{RM}$ obtained at 293 and $343 \mathrm{~K}$ are presented in Figure 4a. The $C(t)$ of ANS in $w_{0}=10 \mathrm{RM}$ at $293 \mathrm{~K}$ shows biexponential decay with time constants of $0.10 \mathrm{~ns}(50 \%)$ and $1.82 \mathrm{~ns}(50 \%)$, respectively (Table 2$)$. The origin of the shorter and longer time components is due to the motion of bulklike water and the interface-bound water of RM, respectively. ${ }^{23}$ With the increase in temperature (Figure 4a), the faster component shows an insignificant change, but the slower component drastically changes indicating that the enhancement of the solvation rate for the interface-bound ANS occurs due to the activation energy barrier crossing transition of bound to free water at the reverse micellar interface. Table 2 shows the corresponding time components of $C(t)$ of ANS in $w_{0}=10$ $\mathrm{RM}$ together with average solvation time, $\left\langle\tau_{\text {solv }}\right\rangle$, at different
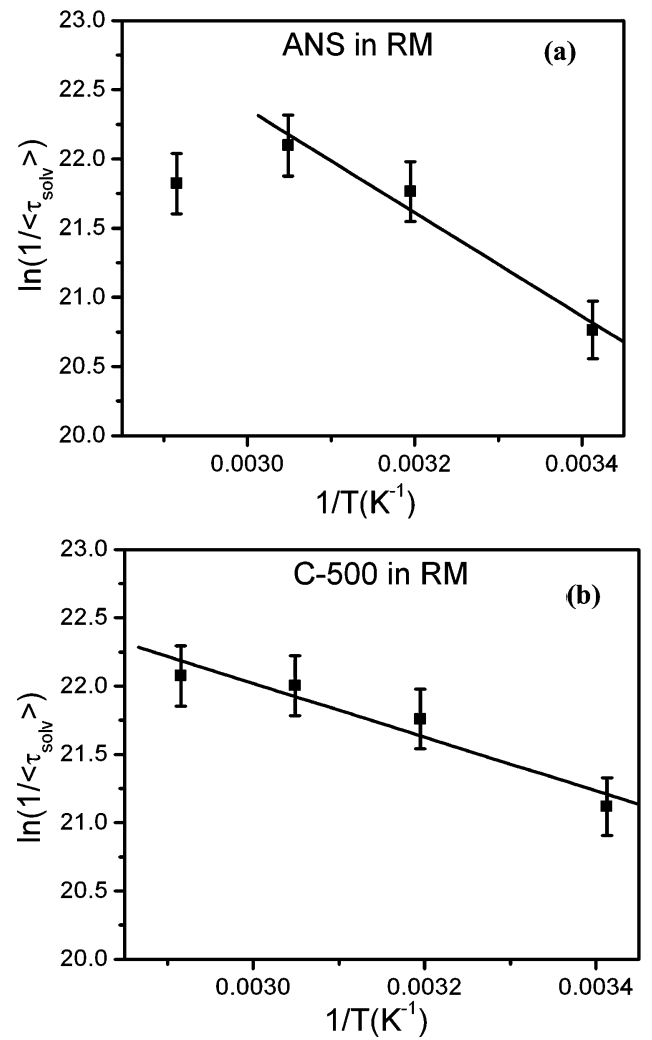

Figure 5. Plot of $\ln \left(1 /\left\langle\tau_{\text {solv }}\right\rangle\right)$ against $1 / T$ for (a) ANS and (b) C-500 in $w_{0}=10$ mixed RM. Solid lines indicate numerical fits to the experimental data points.

temperatures. $\left\langle\tau_{\text {solv }}\right\rangle$ in the system has been calculated using the equation

$$
\left\langle\tau_{\text {solv }}\right\rangle=a_{1} \tau_{1}+a_{2} \tau_{2}
$$

where $a_{1}$ and $a_{2}$ are relative concentrations corresponding to the solvation time constants $\tau_{1}$ and $\tau_{2}$, respectively.

For the probe C-500, selective excitation at $409 \mathrm{~nm}$ has been employed to unravel the dynamics of the trapped interfacial water of RM with the variation of temperature. The red edge excitation wavelength $(409 \mathrm{~nm})$ used in our experiment only excites those populations of C-500 which are in the close vicinity of the water/surfactant interface, which is also evidenced from the earlier literature. ${ }^{29}$ The advantages of red edge excitation has been investigated in the recent literature, ${ }^{44,45}$ which indicates that the red edge excitation of the probe C-500 particularly excites only the interface bound populations. The $C(t)$ curves of C-500 in $w_{0}=10 \mathrm{RM}$ obtained at 293 and $343 \mathrm{~K}$ are presented in Figure $4 \mathrm{~b}$. The $C(t)$ decay of C-500 in $w_{0}=10$ $\mathrm{RM}$ at $293 \mathrm{~K}$ is triexponential with time constants of $0.17 \mathrm{~ns}$ (24.7\%), $1.00 \mathrm{~ns}(63.3 \%)$, and $4.48 \mathrm{~ns}(12.0 \%)$ in contrast to the biexponential decay of ANS in RM at the same temperature. The faster time constants obtained $(0.17$ and $1 \mathrm{~ns})$ are similar to those obtained in the case of ANS in mixed RM. We recognize the slowest time component of $\sim 4.5 \mathrm{~ns}^{46}$ to be the contribution from surfactant head groups, because of the close proximity of the probe $\mathrm{C}-500$ to the interface. The shorter time component $(\sim 0.2 \mathrm{~ns})$ may also be attributed to the fast-moving trapped waters ${ }^{15}$ in the interfacial stern layer of RM. The intermediate time constant of $1 \mathrm{~ns}$ is assigned to bound-type water molecules at the interface ${ }^{47}$ However, the possibility of the contribution of counterions ${ }^{48,49}$ (sodium ions) in the dynamics of bound-type water cannot be completely ruled out. The fitted decay time components of $C(t)$ for C-500 in $w_{0}=10$ 
TABLE 3: Decay Parameters of $C(t)$ of C-500 in $w_{0}=10$ Mixed RM at Different Temperatures

\begin{tabular}{cccrc}
\hline temp $(\mathrm{K})$ & $\tau_{1}\left(\mathrm{a}_{1}\right)(\mathrm{ns})$ & $\tau_{2}\left(\mathrm{a}_{2}\right)(\mathrm{ns})$ & $\tau_{3}\left(\mathrm{a}_{3}\right)(\mathrm{ns})$ & $\left\langle\tau_{\text {solv }}\right\rangle(\mathrm{ns})$ \\
\hline 293 & $0.17(0.25)$ & $1.00(0.63)$ & $4.48(0.12)$ & 0.67 \\
313 & $0.10(0.43)$ & $0.60(0.52)$ & $6.27(0.05)$ & 0.35 \\
343 & $0.13(0.55)$ & $0.58(0.36)$ & $10.25(0.09)$ & 0.28 \\
\end{tabular}

TABLE 4: Decay Parameters of $r(t)$ of ANS in $w_{0}=10$ Mixed RM at Different Temperatures

\begin{tabular}{cllll}
\hline temp $(\mathrm{K})$ & $\tau_{1}\left(\mathrm{a}_{1}\right)(\mathrm{ns})$ & $\tau_{2}\left(\mathrm{a}_{2}\right)(\mathrm{ns})$ & $\tau_{3}\left(\mathrm{a}_{3}\right)(\mathrm{ns})$ & $r_{0}(\mathrm{~ns})$ \\
\hline 293 & $0.11(0.19)$ & $1.35(0.40)$ & $37.90(0.41)$ & 0.38 \\
313 & $0.14(0.35)$ & $1.07(0.31)$ & $35.00(0.34)$ & 0.39 \\
343 & $0.16(0.30)$ & $0.91(0.35)$ & $28.30(0.35)$ & 0.38 \\
\end{tabular}

TABLE 5: Decay Parameters of $r(t)$ of C-500 in $w_{0}=10$ Mixed RM at Different Temperatures

\begin{tabular}{ccccc}
\hline temp $(\mathrm{K})$ & $\tau_{1}\left(\mathrm{a}_{1}\right)(\mathrm{ns})$ & $\tau_{2}\left(\mathrm{a}_{2}\right)(\mathrm{ns})$ & $\tau_{3}\left(\mathrm{a}_{3}\right)(\mathrm{ns})$ & $r_{0}(\mathrm{~ns})$ \\
\hline 293 & $0.10(0.32)$ & $1.17(0.42)$ & $40.47(0.26)$ & 0.38 \\
313 & $0.08(0.47)$ & $0.75(0.40)$ & $34.78(0.13)$ & 0.38 \\
343 & $0.10(0.55)$ & $0.67(0.35)$ & $31.82(0.10)$ & 0.36 \\
\end{tabular}

RM with temperature are depicted in Table 3, which shows that the magnitudes of both slower and faster time components decrease gradually with increase in temperature. However, the slowest solvation time constant (from surfactant head groups) is found to increase with increase in temperature. At the elevated temperature, there is a possibility that some population of C-500 molecules interact more with the polar head groups of the surfactant due to the dehydration of the head groups ${ }^{21}$ and thus increase the slowest solvation time constant. As we are interested in exploring the nature of temperature-dependent solvent relaxation, the longest solvation time constant of $\mathrm{C}-500$ has been excluded in the estimation of the $\left\langle\tau_{\text {solv }}\right\rangle$.

The average solvation time constant of C-500 in the mixed $\mathrm{RM},\left\langle\tau_{\text {solv }}\right\rangle$, at higher temperature (Table 3 ) is found to be different from that at $293 \mathrm{~K}$. The total dynamic Stokes shift for both ANS and C-500 is observed to be more than $1000 \mathrm{~cm}^{-1}$ at all the experimental temperatures. The observed Stokes' shift of the C-500 emission in $w_{0}=10 \mathrm{RM}$ is found to be 1690 $\mathrm{cm}^{-1}$. Following Fee and Maroncelli, ${ }^{50}$ one may calculate the amount of solvation missed in a picosecond setup. According to this method, the difference between the emission frequency at time zero $\left(\nu \mathrm{p}_{\mathrm{em}}(0)\right)$ and the absorption frequency $\left(\nu \mathrm{p}_{\mathrm{abs}}\right)$ in a polar solvent is approximately equal to the difference between steady-state frequencies of emission $\left(v^{\mathrm{np}} \mathrm{em}\right)$ and absorption $\left(v^{\mathrm{np}}\right.$ abs $)$ in a nonpolar solvent. So,

$$
v^{\mathrm{p}}{ }_{\mathrm{em}}(0)=v_{\mathrm{abs}}^{\mathrm{p}}-\left[v_{\mathrm{abs}}^{\mathrm{np}}-v_{\mathrm{em}}^{\mathrm{np}}\right]
$$

With the use of isooctane as the nonpolar solvent, $\nu_{\mathrm{em}}^{\mathrm{p}}(0)$ for $\mathrm{C}-500$ is calculated to be $21212 \mathrm{~cm}^{-1}$. From our time-resolved data for C-500 in $w_{0}=10 \mathrm{RM}, \nu \mathrm{p}_{\mathrm{em}}(0)$ and $\nu \mathrm{p}_{\mathrm{em}}(\infty)$ are found to be 20362 and $18672 \mathrm{~cm}^{-1}$, respectively. The total estimated Stokes' shift is thus $2540 \mathrm{~cm}^{-1}$ compared to the observed Stokes' shift of $1690 \mathrm{~cm}^{-1}$. Thus, in our picosecond setup, we have missed $33 \%$ of the total dynamic spectral shift, which closely matches with that obtained by Sen et al. ${ }^{51}$

Figure 5a displays the Arrhenius plot of ANS in RM in the temperature range of 293-343 K. Assuming Arrhenius dependence of the rate constant $\left(1 /\left\langle\tau_{\text {solv }}\right\rangle\right)$, the activation energy $\left(E_{\mathrm{a}}\right)$ has been evaluated from the logarithmic plot of $1 /\left\langle\tau_{\text {solv }}\right\rangle$ against $1 / T$. For ANS, the first three temperatures $(293-328 \mathrm{~K}$ ) follow regular Arrhenius type behavior, whereas a strong deviation is found around $343 \mathrm{~K}$ (Figure 5a). The magnitude of $E_{\mathrm{a}}$ for ANS is obtained as $7.4 \pm 0.5 \mathrm{kcal} \mathrm{mol}^{-1}$. This activation energy is attributed to the barrier crossing transition from bound to free water at the interface of RM, and the value is very close to the difference between water-micelle and water-water hydrogen bond energies $\left(7-8 \mathrm{kcal} \mathrm{mol}^{-1}\right.$ ) reported earlier. ${ }^{52}$ The $E_{\mathrm{a}}$ value $\left(9 \mathrm{kcal} \mathrm{mol}^{-1}\right)$ reported by Sen et al. ${ }^{9}$ for the hydrophilic probe 4-AP located at the palisade layers of the SDS micelles is also consistent with our results. Figure 5b represents the Arrhenius plot of C-500 in RM. It is evident from the figure that the data agree well with the activation energy barrier model (governed by the Arrhenius equation) for the dynamical equilibrium between bound and free-type water molecules. The corresponding $E_{\mathrm{a}}$ value of $3.9 \pm 0.5 \mathrm{kcal} \mathrm{mol}^{-1}$ is close to the value of
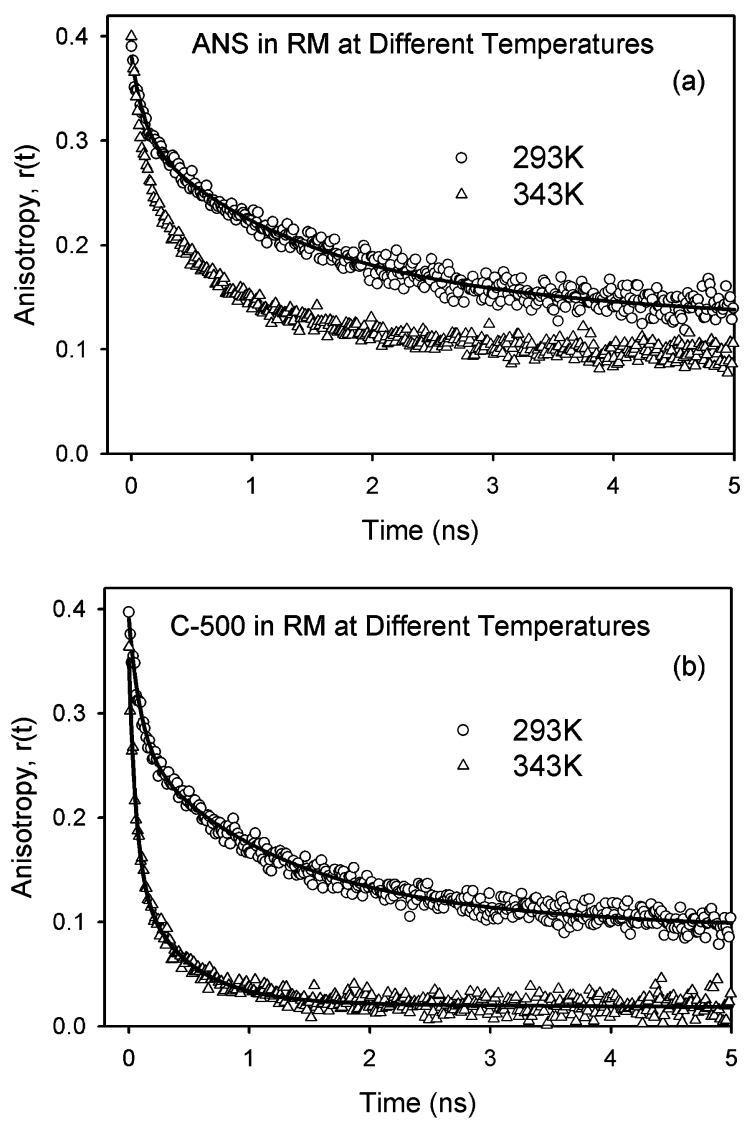

Figure 6. Fluorescence anisotropy decays, $r(t)$, of (a) ANS and (b) C-500 in $w_{0}=10$ mixed RM at 293 and $343 \mathrm{~K}$. 

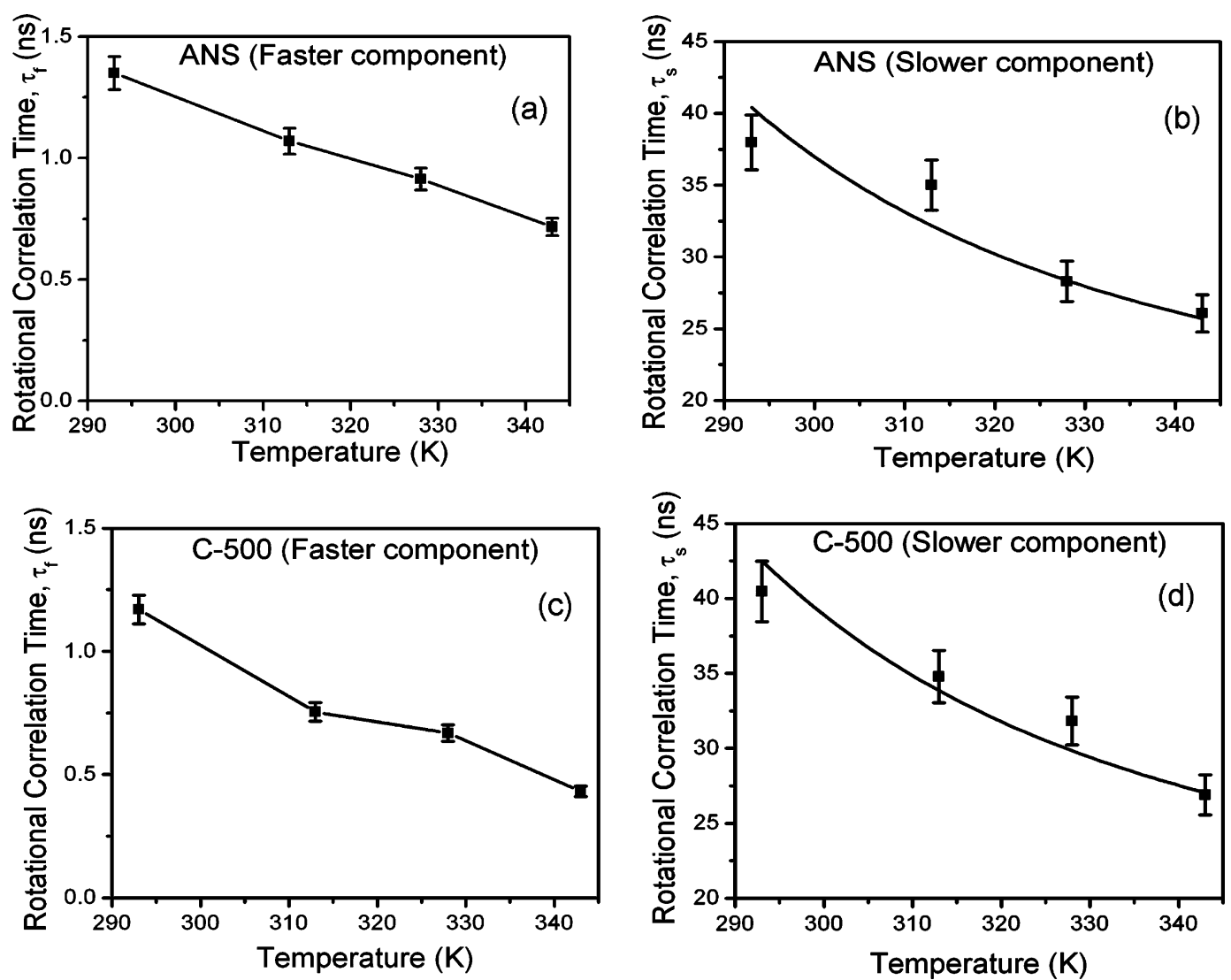

Figure 7. Plots of faster $(\sim 1.5 \mathrm{~ns})$ and slower $(\sim 30 \mathrm{~ns})$ rotational correlation times against temperature for ANS $(\mathrm{a}, \mathrm{b})$ and C-500 (c, d) in $w_{0}=$ 10 mixed RM. Solid lines in (a) and (c) are guides to the eye. Solid lines in (b) and (d) indicate numerical fitting to the experimental data points following the Stokes-Einstein-Debye model.

2.4-4 $\mathrm{kcal} \mathrm{mol}^{-1}$ obtained from molecular dynamic simulation study by Pal et al..$^{52}$ In this study, it is revealed that the interfacial water are of three types, viz., IB1, IB2, and IFW, where IB1 and IB2 water molecules are singly and doubly hydrogen bonded to the head group oxygen atom of the micelle while IFW water molecules are not hydrogen bonded to head groups but to other water molecules. The three species reside in dynamic equilibrium with each other and with bulk water. This study also reveals that IB1 and IB2 are more stable than IFW species with a difference in state energy of $2.4 \mathrm{kcal} \mathrm{mol}^{-1}$, which is comparable to our estimated value of $E_{\mathrm{a}}\left(3.9 \pm 0.5 \mathrm{kcal} \mathrm{mol}^{-1}\right)$ for the probe C-500. The activation energy $\left(E_{\mathrm{a}}\right)$ values of 7.4 and $3.9 \mathrm{kcal} \mathrm{mol}^{-1}$ obtained for the probes ANS and C-500, respectively, also indicate that the proximity of ANS to the RM interface is comparatively lower than that of the probe C-500. The probe ANS essentially reports the overall bound type water to free type water transition in the mixed RM, which has been reported $^{52}$ to be $7-8 \mathrm{kcal} \mathrm{mol}^{-1}$. On the other hand, C-500 in the interface of RM essentially reports the transition of different kinds of interfacial-bound water (IB1, IB2, and IFW) to free water with an activation energy barrier ${ }^{52}$ of $\sim 4 \mathrm{kcal} \mathrm{mol}^{-1}$. Thus, from our study, it is evident that the probe location is extremely important to follow the barrier crossing transition in the reverse micellar water pool.

We rationalize the deviation from the activation energy barrier crossing dynamics as reported by the probe ANS in the RM at $343 \mathrm{~K}$ as follows: It is known that the initial location of the probe C-500 (at $293 \mathrm{~K}$ ) is in the close vicinity of the reverse micellar interface. This enables us to obtain the slowest component (corresponding to solvation from head groups) widely separated from other two faster solvation time constants (free-type water and interface-bound water) at all temperatures.
Hence, in the calculation of the average solvation time constant for C-500, the contribution of solvation from the head group of surfactant can be easily discarded, giving the solvation contribution from solvent molecules alone. Hence, as expected, the probe C-500 follows the Arrhenius model well shown in Figure 5b as the spatial heterogeneity of the probe C-500 is handled analytically. However, for the probe ANS, the situation is slightly different. The ANS molecules in RMs at $293 \mathrm{~K}$ are distributed in a range of locations in the RM interface. Hence, the solvation of ANS reveals shorter and longer time components corresponding to bulklike water and the interface-bound water of RM, respectively. At higher temperatures, when the dehydration of the head group takes place, the contribution to the solvation from the head group also comes into play, which is convoluted with the contribution from interface-bound water of RM. ${ }^{21}$ Due to the incapability of separating the contribution of head group from interface-bound water, the average solvation time constant gives a value which does not fit with the Arrhenius equation depicting the transformation of interfacial bound water molecules to free-type molecules. Thus, we can conclude that the spatial heterogeneity in the location of ANS in RM, which becomes prominent at $343 \mathrm{~K}$ due to the proximity of a certain population of the probes to the surfactant head group, is responsible for the deviation.

Time-resolved fluorescence anisotropy decay, $r(t)$, of ANS and C-500 in RM, measured at their corresponding emission peaks at 293 and $343 \mathrm{~K}$ is depicted in Figure 6a,b. For all the studied temperatures, $r(t)$ is characterized by a slow decay with a considerable offset. The decays fit well triple-exponentially, and the rotational time constants at different temperatures for ANS and C-500 in $w_{0}=10 \mathrm{RM}$ are listed in Tables 4 and 5, respectively. The rotational time constants reflect the temper- 
ature effect on the local environment around the probes. The anisotropy decays for both the probes in quarternary microemulsions are significantly slower than in bulk water. For ANS, $r(t)$ shows decay with relaxation time constants of $0.11 \mathrm{~ns}(19 \%)$, $1.35 \mathrm{~ns}(40 \%)$, and $37.9 \mathrm{~ns}(41 \%)$ at $293 \mathrm{~K}$ (Table 4). The faster relaxation time constant of $\sim 0.1$ ns does not show regular temperature dependence and can be recognized as inertial/ wobbling motion of the probe. ${ }^{53}$ The time constant $\sim 1.5 \mathrm{~ns}$ in the anisotropy decay shows reasonably good temperature dependence and may be due to the rotation of the excited-state species of the probe ANS in $\mathrm{RM},{ }^{21}$ close to the head group of lecithin. The longer time component ( $\sim 40 \mathrm{~ns})$ depicts the residence of the dye at the reverse micellar interface revealing the overall rotation of the RM. ${ }^{54}$ These results can be interpreted as indicative of the dye residing in a range of locations in the interface. With the increase of temperature, the overall rotational dynamics becomes faster revealing the lability of the microenvironment with temperature. ${ }^{6,21}$ The temperature-dependent rotational time constant of $\sim 1.5 \mathrm{~ns}$ (Figure $7 \mathrm{a}$ ) reveal that the microviscosity around the probe ANS varies from 3.3 to 2.1 $\mathrm{cP}$ in the temperature range 293-343 K using the StokesEinstein-Debye (SED) equation. According to this equation, rotational relaxation time $\left(\tau_{\mathrm{r}}\right)$ is defined as

$$
\tau_{\mathrm{r}}=\frac{\eta V}{k_{\mathrm{B}} T}
$$

where $V$ is the solute volume and $\eta$ is the shear viscosity coefficient of the solvent. The value of the microviscosity near room temperature is in close agreement with the reported value $(\sim 5 \mathrm{cP})$ in AOT RM. ${ }^{55}$ On the other hand, temperature dependence of the slower relaxation time as shown in Figure $7 \mathrm{~b}$ can be fitted using the SED equation (solid line), revealing the average diameter of the RM to be $8.9 \mathrm{~nm}$, in good agreement with DLS measurement $(\sim 9.2 \mathrm{~nm})$. The temperature-dependent viscosity coefficients of isooctane have been obtained from the literature. ${ }^{56}$ Similar to ANS, the anisotropy of the probe C-500 displays triple-exponential decay with time constants of $0.1 \mathrm{~ns}$ (32\%), $1.2 \mathrm{~ns}$ (42\%), and $40.5 \mathrm{~ns}(26 \%)$ (Table 5). The faster and slower rotational time constants (i.e. $\tau_{1}$ and $\tau_{2}$ in Table 5) obtained for the probe C-500 in $w_{0}=10 \mathrm{RM}$ at different temperatures are in good agreement with those reported by Mitra et al. ${ }^{6}$ in the AOT RM system. The decrease in the slower time constant of C-500 in $w_{0}=10 \mathrm{RM}$ (i.e $\tau_{2}$ in Table 5) with increasing temperature is an indication of the probe becoming labile at elevated temperature. The microviscosity around the probe C-500 is found to vary from 4.3 to $1.9 \mathrm{cP}$ at the abovementioned temperature range (Figure 7c), which is consistent with the values reported in literature. ${ }^{55}$ As shown in Figure 7d, the slower component of the anisotropy decay of C-500 in RM also follows the SED model, revealing the similar diameter $(9.0$ $\mathrm{nm}$ ) of the RM obtained from DLS studies.

\section{Conclusion}

This study explores the validity and divergence of the activation energy barrier-crossing model for bound to free type water transition to explain the temperature-dependent solvation dynamics of the probes ANS and C-500 at the interfacial region of AOT/lecithin mixed RM within its overall structural integrity. With the increase in temperature, the environmental dynamics reported by C-500 in the mixed RM become progressively faster and follow the Arrhenius equation in our experimental temperature range 293-343 K. However, ANS in the mixed RM follows the Arrhenius equation in the temperature range 293-
$328 \mathrm{~K}$ and shows a deviation from the Arrhenius equation at $343 \mathrm{~K}$. The deviation from the Arrhenius equation for ANS in $\mathrm{RM}$ at $343 \mathrm{~K}$ is attributed to increasing contributions from the motions of the surfactant head groups of RMs due to the transformation of interfacial bound water molecules to freetype molecules. The activation energy values, $E_{\mathrm{a}}$, obtained from our study for both the probes, ANS and C-500, are in good agreement with the MD simulation results reported earlier. Our studies clearly point out that the location of the probe is extremely important to obey the Arrhenius equation.

Acknowledgment. S.S.N. and R.S. thank the CSIR and UGC of India, respectively, for fellowships. We thank the DST of India (SR/FTP/PS-05/2004) for a financial grant.

\section{References and Notes}

(1) Fukuzaki, M.; Miura, N.; Shinyashiki, N.; Kurita, D.; Shioya, S.; Haida, M.; Mashimo, S. J. Phys. Chem. 1995, 99, 431.

(2) Jordanides, X. J.; Lang, M. J.; Song, X.; Fleming, G. R. J. Phys. Chem. B 1999, 103, 7995.

(3) Marchi, M.; Sterpone, F.; Ceccarelli, M. J. Am. Chem. Soc. 2002, $124,6787$.

(4) Teeter, M. M.; Yamano, A.; Stec, B.; Mohanty, U. Proc. Natl. Acad. Sci. U.S.A. 2001, 98, 11242. 2013.

(5) Nandi, N.; Bhattacharyya, K.; Bagchi, B. Chem. Rev. 2000, 100,

(6) Mitra, R. K.; Sinha, S. S.; Pal, S. K. Langmuir 2008, 24, 49. 7174

(7) Hara, K.; Kuwabara, H.; Kajimoto, O. J. Phys. Chem. A 2001, 105,

(8) Shen, D.; Han, B.; Dong, Y.; Chen, J.; Mu, T.; Wu, W.; Zhang, J.; Wu, Z.; Dong, B. J. Phys Chem. B 2005, 109, 5796.

(9) Sen, P.; Mukherjee, S.; Halder, A.; Bhattacharyya, K. Chem. Phys. Lett. 2004, 385, 357 .

(10) Nandi, N.; Bagchi, B. J. Phys. Chem. B 1997, 101, 10954.

(11) Balasubramanian, S.; Pal, S.; Bagchi, B. Phys. Rev. Lett. 2002, 89,115505 .

(12) Kumbhakar, M.; Goel, T.; Mukherjee, T.; Pal, H. J. Phys. Chem. B 2004, 108, 19246.

(13) Kumbhakar, M.; Goel, T.; Nath, S.; Mukherjee, T.; Pal, H. J. Phys Chem. B 2006, 110, 25646.

(14) Mitra, R. K.; Sinha, S. S.; Pal, S. K. J. Phys. Chem. B 2007, 111, 7577

(15) Sarkar, N.; Das, K.; Datta, A.; Das, S.; Bhattacharyya, K. J. Phys. Chem. 1996, 100, 10523.

(16) Dokter, A. D.; Woutersen, S.; Bakker, H. J. Proc. Natl. Acad. Sci. U.S.A. 2006, 103, 15355 .

(17) Rosenfeld, D. E.; Schmuttenmaer, C. A. J. Phys. Chem. B 2006 , 110,14304

(18) Willard, D. M.; Riter, R. E.; Levinger, N. E. J. Am. Chem. Soc. 1998, 120,4151 .

(19) Riter, R. E.; Willard, D. M.; Levinger, N. E. J. Phys. Chem. B 1998, 102, 2705.

(20) Nucci, N. V.; Vanderkooi, J. M. J. Phys. Chem. B 2005, 109, 18301.

(21) Banerjee, D.; Sinha, S. S.; Pal, S. K. J. Phys. Chem. B 2007, 111, 14239

(22) Paul, B. K.; Mitra, R. K. J. Colloid Interface Sci. 2005, 288, 261.

(23) Zhang, J.; Bright, F. V. J. Phys. Chem. 1991, 95, 7900.

(24) Simmons, B.; Agarwal, V.; McPherson, G.; John, V.; Bose, A. Langmuir 2002, 18, 8345.

(25) Simmons, B. A.; Li, S.; John, V. T.; McPherson, G. L.; Bose, A.; Zhou, W.; He, J. Nano Lett. 2002, 2, 263.

(26) Horng, M. L.; Gardecki, J. A.; Papazyan, A.; Maroncelli, M. J. Phys. Chem. 1995, 99, 17311.

(27) Lakowicz, J. R. Principles of fluorescence spectroscopy; Kluwer Academic/Plenum: New York, 1999.

(28) O'Connor, D. V.; Philips, D. Time correlated single photon counting; Academic Press: London, 1984.

(29) Majumder, P.; Sarkar, R.; Shaw, A. K.; Chakraborty, A.; Pal, S. K. J. Colloid Interface Sci. 2005, 290, 462.

(30) Nad, S.; Pal, H. J. Phys Chem. A 2003, 107, 501

(31) Das, K.; Jain, B.; Gupta, P. K. Chem. Phys. Lett. 2005, 410, 160.

(32) Das, K.; Jain, B.; Patel, H. S. J. Phys. Chem. A 2006, 110, 1698.

(33) Zhao, G.-J.; Han, K.-L. J. Phys. Chem. A 2007, 111, 2469.

(34) Das, S.; Thomas, K. G.; Ramanathan, R.; George, M. V.; Kamat,

P. V. J. Phys. Chem. 1993, 97, 13625.

(35) Zhao, G.-J.; Han, K.-L. J. Phys. Chem. A 2007, 111, 9218.

(36) Zhao, G.-J.; Liu, J.-Y.; Zhou, L.-C.; Han, K.-L. J. Phys. Chem. B 2007, 111, 8940. 
(37) Zhao, G.-J.; Han, K.-L. J. Chem. Phys. 2007, 127, 024306.

(38) DeToma, R. P.; Easter, J. H.; Brand, L. J. Am. Chem. Soc. 1976, 98,5001 .

(39) Kosower, E. M. Acc. Chem. Res. 1982, 15, 259.

(40) Zhong, D.; Pal, S. K.; Zewail, A. H. ChemPhysChem 2001, 2, 219.

(41) Dutt, G. B. J. Phys. Chem. B 2002, 106, 7398.

(42) Kumbhakar, M.; Mukerjee, T.; Pal, H. Photochem. Photobiol. 2005, $81,588-594$

(43) Pal, S. K.; Peon, J.; Zewail, A. H. Proc. Natl. Acad. Sci. U.S.A. 2002, 99, 15297.

(44) Sen, P.; Ghosh, S.; Sahu, K.; Mondal, S. K.; Roy, D.; Bhattacharyya, K. J. Chem. Phys. 2006, 124, 204905.

(45) Mandal, U.; Adhikari, A.; Dey, S.; Ghosh, S.; Mondal, S. K.; Bhattacharyya, K. J. Phys. Chem. B 2007, 111, 5896.

(46) Faeder, J.; Ladanyi, B. M. J. Phys. Chem. B 2005, 109, 6732.

(47) Bhattacharyya, K. Acc. Chem. Res. 2003, 36, 95.
(48) Kumbhakar, M.; Goel, T.; Mukherjee, T.; Pal, H. J. Phys. Chem. B 2005, 109, 14168.

(49) Huppert, D.; Ittah, V.; Kosower, E. M. Chem. Phys. Lett. 1989, 159, 267.

(50) Fee, R. S.; Maroncelli, M. Chem. Phys. 1994, 183, 235.

(51) Sen, S.; Dutta, P.; Sukul, D.; Bhattacharyya, K. J. Phys. Chem. A 2002, 106, 6017

(52) Pal, S.; Balasubramanian, S.; Bagchi, B. J. Phys. Chem. B 2003 107,5194

(53) Myers, A. B.; Pereira, M. A.; Holt, P. L.; Hochstrasser, R. M. J. Chem. Phys. 1987, 86, 5146.

(54) Shaw, A. K.; Sarkar, R.; Banerjee, D.; Hintschich, S.; Monkman, A.; Pal, S. K. J. Photochem. Photobiol., A: Chem. 2007, 185, 76.

(55) Corbeil, E. M.; Riter, R. E.; Levinger, N. E. J. Phys. Chem. B 2004 108, 10777.

(56) Padua, A. A. H.; Fareleira, J. M. N. A.; Calado, J. C. G. J. Chem. Eng. Data 1996, 41, 1488. 